\title{
A project in re-inforcing the effectiveness of the primary care team in the area of mental health
}

\author{
Maria Rosen, Locum Consultant Psychotherapist, Hackney Hospital, London E9 6BE
}

It seems that increasingly patients are being referred to primary care teams and GPs in health centres rather than to psychiatrists in a hospital setting. With the growth in the number of patients being maintained in the community, it is becoming more important to pay attention to this sphere and to aid primary care teams to be clinically effective. It was from this perspective that I became involved as facilitator to a primary care team on a one year project.

The following is a description of this project, of the difficulties encountered, and the outcome. I hope my description may be of use to other facilitators, for whom I am arguing there is a growing role.

The request was for me to facilitate a weekly staff group meeting set aside for case discussions, as the meetings had become apathetic and stale. It was hoped I would facilitate a process of change where there would be developments in the work instead of stagnation.

The primary care team at the health centre consisted of GPs, social workers, health visitors, nurses (including CPNs) and a part-time psychotherapist doing some long-term psychotherapy. Most of the care team used a brief therapy model, whether individual or family. The staff varied in their level of expertise and training in psychotherapy from the relatively inexperienced to the experienced.

\section{Description of the staff meeting}

The meeting was held for one-and-a-half hours weekly, chaired by the rotating chairman and was to be facilitated by myself. It was a large group of, at times, 30 people including the primary care team and occasional visitors. During the first few weeks of these meetings, the apathy about which members had complained was noteworthy. At the same time it appeared there was a strong expectation and hope that I would rapidly effect some transformation of the situation. This did not happen. However, I did begin to gradually identify certain inhibiting factors and feed this back to the group members.

\section{Identified factors and problems stalemating the meeting}

Large group phenomena and its influence

In this group, large group phenomena were present. There was a high degree of passivity and members found it difficult to speak out. There was a lot of dependency and idealisation with hopes of the facilitator solving all the problems. There was, however, very little feeling of a working group as had been reported initially.

\section{Idealisation denigration axes}

The mood of the group often expressed hopes of solution from the outside. There was unawareness of solutions being found by members of the group. Denigration of the group was obvious in terms of people leaving early to go to other group meetings. The idea that staff were easily replaceable was sometimes present, in that people did not seem to know when a staff member was leaving, e.g. at retirement. These kinds of attitudes also made the concept of on-going work difficult.

\section{Trainer/trainee phenomena}

Quite often a 'trainee' would experience difficulty in case presentation. There was a high awareness of hierarchy in the group and some trainees experienced this as very inhibiting. One solution was to go off to their own separate discussion or supervision group at another institution. In this way some splitting was occurring: the large staff group was seen as "not good", while the separate supervision group was "good". A lot of testing occurred around this issue and in later months, more work was brought to this meeting. The staff group became seen to be good enough to bring work for supervision.

\section{Lack of continuity of casework, of on-going work}

There was little sense of continuity about casework. Feelings of failure and of hopelessness were openly expressed about some of the 'difficult problems of individuals and families'. Feelings of frustration were gradually expressed by members about not knowing what happened to cases which had been considered in previous weeks. After discussion about this problem the structure of the group was changed by democratic decision to include a structured follow-up time within the group. This led to greater involvement of members and much on-going work. It was now more of a work-group in Bion's terms (1961). 


\section{Unexpressed rivalry in different sab-groups}

A strong covert rivalry between different sub-groups gradually emerged. This was being expressed in a very negative way and deadening the group. Some GPs absented from the meetings, or left early. Once a month nurses went to their separate 'nurses' meeting'. One sub-group of staff who worked with older patients, absented themselves emotionally by never bringing any cases but sitting silently throughout the group. It was only gradually that this pattern of absenting in one way or another became obvious.

Thus, there was a feeling of 'groupiness' within the group, with rival interests and underlying fears that different groups were only interested in themselves. A certain intensity and level of trust had to be reached before these feelings could be expressed. I was used as a safe witness with whom to ventilate such communications and feelings. Also, coming from 'outside', I was seen as having no particular bias or affiliation with any particular sub-group inside. The group used me as a safe container for these intense group rivalries. Once expressed, there was a fuller participation and exchange between members, making room for more friendly working feelings and a true work group.

\section{The paranoid element}

At several stages there was an increase in paranoid feelings in the group and people became less willing to use it. This was particularly in evidence following a meeting in which there were a number of visitors, some of whom appeared unannounced and had to be identified. There was an atmosphere of chaos and flooding at that meeting and hardly any real discussion took place. Many people voiced worries about confidentiality and also said they felt overwhelmed sometimes by the number of visitors. It became obvious that there was no clear protocol for vetting visitors' reliability about confidentiality and limiting the number of visitors in any one group. With further discussion it became clear that there was a need to introduce a more formalised structure to ensure the boundaries were kept. An organising chairman was then appointed who could be approached during the week concerning visitors. The effect of this development was to increase real work relationships between members of the group, its boundaries were more clearly defined, and it was able to become more of a work group.

\section{Some examples of work in case discussions}

Sometimes cases were presented, obviously for help with referral to other agencies. At other times cases were presented for 'information exchange'. Cases were increasingly presented where case-workers wanted to handle the case more effectively themselves, that is, to provide more effective primary prevention. This involved some kind of brief therapy at regular or irregular intervals. A whole range of problems came under this category, and as the group became more of a work group and there came to exist a setting and structure where such work could occur, staff were enabled to become more aware of defensive operations in patients, such as avoidance, denial, splitting, avoidance of dependency needs, and the key-workers could then take up these relevant issues constructively with their patients.

\section{Range of problems in case discussions}

These included: separation problems, psychosomatic problems, problems of the dying and bereaved, alcoholics, drug addicts, puerperal problems, and abuse problems.

\section{My task as facilitator}

I found my task as facilitator encompassed the following:

reliable identifier of difficulties and inhibitory factors within the group as non-partisan facilitator, I was safe to ventilate anxieties and rivalries, allowing group members to reach a more objective view

builder and maintainer of boundaries of the group which had previously been much too fluid for constructive work.

\section{Comment}

The difficulties of the staff group were such that the level of functioning was rather low and seen to be so by the members. In Bion's (1961) terms there was very little presence of a work group in evidence. He stated that the work group was "constantly perturbed by influences which come from other group phenomena". My function was to find, observe and identify such disturbing influences and feed these observations back to the staff group. Group members were then gradually able to deal with these other group phenomena so clearing the way for more effective work. There was an increase in the number of patients being treated, for example, a group was started for patients able to use group therapy and there was a new project launched to give certain addictive patients therapy. Staff members of all disciplines became increasingly confident and competent in their brief individual therapy and 
family therapy and effectively using the group for supervision.

It would also appear that this was a cost-effective use of a psychiatrist/psychotherapist's time, in that for one-and-a-half hours per week 30 health care staff were able to have supervision and the numbers of patients with psychological problems treated by the primary care team over the year increased. I have attempted to describe the process of increasing the effectiveness of the staff group in this paper and hope this may be useful for facilitators in other primary care teams.

\section{Acknowledgements}

This work was done under the auspices of the Tavistock Clinic. I wish to thank especially $\mathrm{Dr}$ Stanford Bourne for comments and discussion of this paper.

\section{Reference}

Bion, W. R. (1961) Experiences in Groups. London: Tavistock Publications, p. 129.

\title{
Improving psychiatric day hospital attendance - a Liverpool intervention study
}

\author{
G. T. Ridley, Registrar, Mersey Regional Psychiatry Training Scheme; K. Clare, \\ Charge Nurse; M. E. DEwEY, Lecturer in Psychological Statistics; and I. A. DAvidson, \\ Consultant Psychiatrist, University Department of Psychiatry, Royal Liverpool \\ Hospital, Liverpool L7 8XP
}

In spite of the somewhat disordered development of day care for the mentally ill, the Psychiatric Day Hospital (PDH) has become accepted as an essential element in the mental health services. Several recent studies have defined a role for the PDH in the management of more acute psychiatric disorders (Creed et al, 1989), and this is likely to become increasingly important with the continuing reduction in the availability of in-patient facilities.

An area which has received relatively little attention in the literature, but which is likely to become increasingly important with greater reliance on day hospital care, is that of a poor overall attendance rate. Few studies address this issue directly, but, defining non-attendance as failure to attend beyond the first day of admission, studies of consecutive PDH referrals report rates of roughly $50 \%$, while those including some form of post-referral screening report rates nearer to $20 \%$ (Dick, 1986). It therefore seems possible that poor attendance at PDH may be improved by a relatively simple intervention, and it is the purpose of this study to address this issue specifically.

\section{The study}

The Royal Liverpool Hospital PDH is a 40-place unit on the second floor of a large teaching hospital, adjacent to the university department of psychiatry and two wards, and serving an inner city area containing the most underprivileged wards in Liverpool.

Staff consists of a charge nurse, three staff nurses, a senior occupational therapist, an occupational therapy assistant, and a full-time registrar. Characteristics of the day patient population are similar to those nationally (Conway et al, 1988).

Non-attendance rate was estimated in 1988 to be roughly $50 \%$, and, in an effort to improve on this, an assessment package was introduced in early 1989 , comprising a semi-structured interview by a senior nurse, a visit to the unit and a booklet describing its activities. The effects of this were studied by examining case notes of consecutive referrals to the unit eight months pre-intervention and eight months post-intervention, for information on age, sex, DSM-III diagnosis, employment and marital status, attendance, and time between referral and admission. Figures were collected separately for those attending by ambulance and those travelling by other means.

No changes were made in the availability or range of specific treatments offered either within or outside the unit during the study period, and referring doctors were unaware that the intervention had been implemented. 